\title{
Exploring Privacy Breaches and Mitigation Strategies of Occupancy Sensors in Smart Buildings
}

\author{
Phillip Lee \\ Honeywell \\ phillip.lee@honeywell.com \\ Sharad Mehrotra \\ University of California, Irvine \\ sharad@ics.uci.edu
}

\author{
Eun-Jeong Shin \\ University of California, Irvine \\ ejshin1@uci.edu
Nalini Venkatasubramanian
University of California, Irvine nalini@ics.uci.edu

\author{
Valerie Guralnik \\ Resideo \\ Valerie.Guralnik@resideo.com \\ Kevin T. Smith \\ Tridium \\ ksmith@tridium.com
}

\begin{abstract}
Occupancy sensors are an integral part of many smart building applications, including energy and space optimizations. However, as more occupancy data is collected at an increasingly fine-grained level, possibilities of potential privacy breaches also increase. In this paper, we propose an attack in which the adversary associates an individual with the occupancy sensor by combining the occupancy data with other public information that could easily be obtained online. We conduct an experiment using real-world data to demonstrate that the proposed attack is not only feasible, but the adversary can efficiently identify a small set of sensor IDs that contains the ID of interest given a relatively small amount of additional information. We also summarize a number of mitigation strategies against the proposed attack.
\end{abstract}

\section{CCS CONCEPTS}

\section{- General and reference $\rightarrow$ Experimentation.}

\section{KEYWORDS}

IoT, privacy, occupancy, building

\section{ACM Reference Format:}

Phillip Lee, Eun-Jeong Shin, Valerie Guralnik, Sharad Mehrotra, Nalini Venkatasubramanian, and Kevin T. Smith. 2019. Exploring Privacy Breaches and Mitigation Strategies of Occupancy Sensors in Smart Buildings. In The 1st ACM International Workshop on Technology Enablers and Innovative Applications for Smart Cities and Communities (TESCA'19), November 1314, 2019, New York, NY, USA. ACM, New York, NY, USA, 4 pages. https: //doi.org/10.1145/3364544.3364827

\section{INTRODUCTION}

Smart buildings are becoming increasingly prevalent as an integral part of society. Equipped with various sensors and actuators, smart buildings enable automated building controls including light, Heating, Ventilation, and Air Conditioning (HVAC), and space optimization [1]. This results in personalized comfort controls for the

Permission to make digital or hard copies of all or part of this work for personal or classroom use is granted without fee provided that copies are not made or distributed for profit or commercial advantage and that copies bear this notice and the full citation on the first page. Copyrights for components of this work owned by others than ACM must be honored. Abstracting with credit is permitted. To copy otherwise, or republish, to post on servers or to redistribute to lists, requires prior specific permission and/or a fee. Request permissions from permissions@acm.org.

TESCA'19, November 13-14, 2019, New York, NY, USA

(C) 2019 Association for Computing Machinery.

ACM ISBN 978-1-4503-7015-8/19/11 ..\$15.00

https://doi.org/10.1145/3364544.3364827 building occupants as well as economic savings for the owners of buildings. However, as more fine-grained data at individual levels are collected for building automation, dangers of potential privacy breach also increase [2].

One type of sensor that has been identified as a potential privacy risk is the occupancy sensor [3]. Occupancy sensors detect presence of an individual which enables dynamic control of light and HVAC systems based on the detected occupancy information. However, the collected occupancy data could potentially be used for inference of individual's occupancy patterns and tracking of individuals [4].

In this paper, we study privacy implications of occupancy sensors in smart buildings. In particular, we are interested in a setting where each individual is given a primary location where the person is expected to be throughout the day, such as cubicles or offices.

In many cases, the automation control is indifferent to the identities of building occupants. For example, detection of a presence is sufficient to trigger the light to be turned on at the sensed location. The automated building control does not require the knowledge of who is triggering the occupancy sensor, but only requires the information that a presence is detected at a particular location.

For this reason, the database containing user-identifying information is typically kept separately from the database storing occupancy information, and there is no communication between those databases. Because of this separation, it is easy to believe that unless the adversary compromises both databases, it is difficult to infer the mapping between the set of sensors and the identities of individuals. The findings of this paper demonstrate that maintaining separate databases is insufficient for mitigating privacy leakage from occupancy sensors.

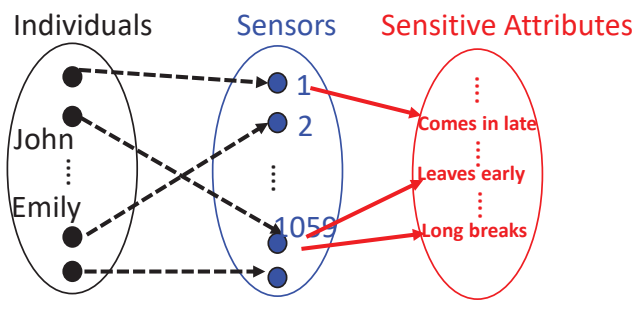

Figure 1: Figure illustrating the privacy implications of the presence data.

Specifically, we make the following set of contributions.

(1) We propose a new privacy breaching attack in smart buildings when the gathered occupancy information does not 
contain unique identifiers of individuals. In the proposed attack, the adversary combines the sensed occupancy data with auxiliary information which is often public. This attack enables the adversary to identify the sensor ID whose location is associated with the individual, which in turn, leads to leakage of sensitive information (Figure 1).

(2) We perform an experiment using real-world data to validate the feasibility of the proposed attack. The experiment shows that even with a small set of auxiliary information, the adversary can obtain a significant amount of information regarding the sensor ID at the primary location of the targeted individual.

(3) We propose a set of mitigation strategies against the proposed attack including the use of differential privacy and time-based encryption and deletion of the stored occupancy data.

\section{RELATED WORK}

Use of occupancy sensors for various building applications has been a subject of active research for both academia and industry [3, 5-8]. Occupancy-driven building applications include both energy [5] as well as space optimization [7]. As occupancy data is being widely implemented, identifying privacy leakage and developing potential mitigation strategies against the potential privacy breaches have been gaining increasing attention $[3,4]$.

In [3], an optimization framework was proposed where the goal is to minimize the information regarding the location traces of individuals while guaranteeing performance of the HVAC system. However, the paper starts with the assumption that the mapping between the locations and individuals are available to the adversary.

A differentially private mechanism for streaming data was studied in [9], and the proposed mechanism was implemented on the building occupancy data in [10]. In this work, differential privacy ensures that an occupancy pattern in a particular zone is statistically similar whether an individual is in the zone or not. In this paper, we study the problem of linking an individual and the sensor ID at each presence sensor level.

\section{MODEL AND PRELIMINARIES}

In this section, we present the adversary and building models. We also define the notations that will be used throughout the paper.

\subsection{Building Model}

We consider a building that is divided into multiple locations where each location is equipped with a presence sensor. We assume that a presence sensor is capable of inferring whether or not the location is currently occupied, but is not capable of inferring which specific individual is triggering the occupancy. An example of such a sensor would be a motion detector which uses ultrasonic or microwave technologies. Each individual is given a primary location where the individual will be spending most of the time when the individual is in the building. For example, a primary location might be a designated office, a particular room, or a particular cubicle.

The set of presence sensors are denoted as $\mathcal{S}$. Each sensor periodically detects presence in its neighborhood. The occupancy pattern of sensor $s_{j} \in \mathcal{S}$ at time $t$ is denoted as $o_{j}^{t}$ and is defined as

$$
o_{j}^{t}= \begin{cases}1, & \text { if sensor } s_{j} \text { detects presence at time } t \\ 0, & \text { else. }\end{cases}
$$

Similarly, we define $o_{j}^{\left[t_{0}, t_{f}\right]}=1$ if occupancy is continuously detected during the time interval $\left[t_{0}, t_{f}\right]$ and $o_{j}^{\left[t_{0}, t_{f}\right]}=0$ otherwise.

\subsection{Adversary Model}

Any application that consumes the occupancy data could potentially use the data other than its intended purposes. The goal of the adversary is to infer the sensor ID that is installed at the primary location of the targeted individual $i$ denoted as $s_{i}$. It is assumed that the adversary can query any sensor ID to observe the presence information at any given time $t$.

The adversary also has a set of auxiliary information of the individual $i$, which is denoted as $\mathcal{A}_{i}$. Auxiliary information is defined as occupancy-pattern information that the adversary has about the individual. An example of a piece of auxiliary information is a travel schedule of the individual from which the adversary can infer that the individual was not in the building for a given time interval. Such information is often public information that can easily be obtained from social network sites including LinkedIn ${ }^{1}$, where postings may include an individual receiving an award or giving a presentation at a conference. Such information would indicate that the individual was not present in the building on the days when this was posted.

For each auxiliary information $a_{i}^{\left[t_{0}, t_{f}\right]} \in \mathcal{A}_{i}$, we denote $a_{i}^{\left[t_{0}, t_{f}\right]}=$ 1 if the individual $i$ was known to be in the building during the time interval $\left[t_{0}, t_{f}\right]$ and $a_{i}^{\left[t_{0}, t_{f}\right]}=0$ if the individual $i$ was known to be absent from the building during the time interval $\left[t_{0}, t_{f}\right]$.

\section{PROPOSED ATTACK}

In this section, we propose an attack in which the adversary infers the sensor ID of the targeted individual $i$ with high probability by combining the auxiliary information with the queried occupancy patterns. The main idea of the attack is to exploit the fact that the sensor $s_{i}$ has to satisfy all the conditions presented by the auxiliary information $\mathcal{A}_{i}$. An illustration of the proposed attack is shown in Figure 2.

Without loss of generality, we index the set $\mathcal{A}_{i}$ as

$$
\mathcal{A}_{i}=\left\{a_{i}^{\left[t_{0}, t_{1}\right]}, \ldots, a_{i}^{\left[t_{n-1}, t_{n}\right]}\right\}
$$

$\mathcal{A}_{i}$ represents the set of auxiliary information obtained by the adversary for individual $i$. For each item of auxiliary information $a_{i}^{\left[t_{m-1}, t_{m}\right]} \in \mathcal{A}_{i}$, define the set $\mathcal{S}_{m}^{i} \subset \mathcal{S}$ as

$$
\mathcal{S}_{m}^{i}=\left\{s_{j}: o_{j}^{\left[t_{m-1}, t_{m}\right]}=a_{i}^{\left[t_{m-1}, t_{m}\right]}\right\}
$$

In other words, the set $\mathcal{S}_{m}^{i}$ is the set of sensors such that the corresponding occupancy patterns are consistent with the auxiliary information $a_{i}^{\left[t_{m-1}, t_{m}\right]}$. Given $n$ auxiliary information, the adversary can construct $n$ such sets, and the sensor $s_{i}$ has to be in all of the sets $\mathcal{S}_{1}^{i}, \ldots, \mathcal{S}_{n}^{i}$. Assuming the adversary does not have any a priori

\footnotetext{
${ }^{1}$ https://www.linkedin.com/
} 
information regarding $s_{i}$, the probability of correctly guessing $s_{i}$ given $\mathcal{A}_{i}$ is written as

$$
\mathrm{P}\left(\hat{s}_{i}=s_{i} \mid \mathcal{A}_{i}\right)=\frac{1}{\left|\bigcap_{m=1}^{n} \mathcal{S}_{m}^{i}\right|}
$$

where $\hat{s}_{i}$ is the adversary's estimate of $s_{i}$.

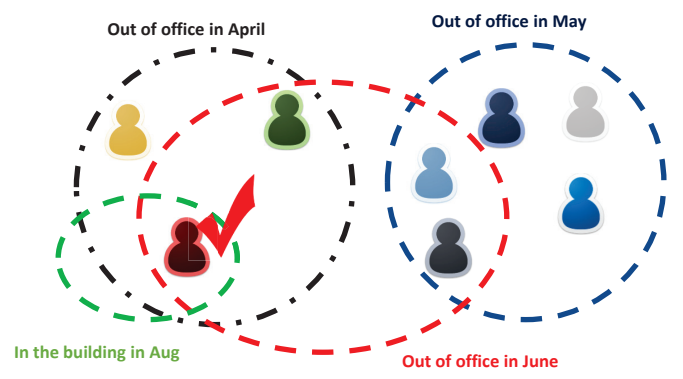

Figure 2: Figure illustrating the proposed attack.

\section{EXPERIMENTAL STUDY}

We conducted an experimental study using real-world occupancy sensor data from an office building.

We selected one zone of the office building for the experimental study which contained 1663 motion detectors. Each motion detector records a binary value of 1 if it senses presence via motion and 0 otherwise. The presence value is recorded every 5 seconds and transmitted to a centralized database. The auxiliary information we considered was daily travel schedules of individuals. The travel schedules used for this study were obtained either with user consent or through public data from Linkedin. The set of auxiliary information was gathered within the period of 5 months.

For each day, we assumed that an individual was present in the building if person stayed in his or her primary location for more than an hour, which is equivalent to having more than 720 number of 1 's in the presence data for one day. The assumption was made to account for triggering of the motion detector by bypassing people or patrolling security guards at night time. If the presence data for a sensor contained less than 720 number of 1's for one day, then we assume that the area covered by the sensor was unoccupied for that day. We conducted our experiment on four employees. For the first case, we used three pieces of auxiliary information.

(1) The person traveled for two days during weekdays and therefore was not present in the building during the travel.

(2) The person has not worked during the weekends for a particular month.

(3) The person returned to work after two days of travel and was present in the building on the third day.

Without any additional information, the probability of correctly guessing the sensor ID that is located at the person's primary location is $\frac{1}{1663}$. After identifying the set $\mathcal{S}_{1}$ that satisfied the first auxiliary information 1), the cardinality of the set was reduced to 14 from 1663. In addition, after obtaining the intersection $\mathcal{S}_{1} \cap \mathcal{S}_{2} \cap \mathcal{S}_{3}$, where $\mathcal{S}_{2}$ and $\mathcal{S}_{3}$ are the sets that satisfied the auxiliary information
2) and 3) respectively, the cardinality of the new set was reduced to 9 from 1663 . We have verified that the person's primary location (an office space) was indeed one of the nine candidate locations that we identified.

Figure 3 shows how probabilities of correctly identifying primary locations (Equation (3)) change with auxiliary information for all four cases. The horizontal axis is the relative time of the year when the auxiliary information was gathered within the period of five months. For cases 2,3, and 4, the off label shown in Figure 3 indicates that the person was not present in the building during weekdays and the on label indicates that the person was present in the building. In all four cases, we observed that with two to three pieces of auxiliary information, the cardinalities of candidate sets will reduce to approximately 10 , resulting in significant increase of probabilities of correctly identifying the primary locations for all four individuals to approximately 0.1 from $\frac{1}{1663}$.

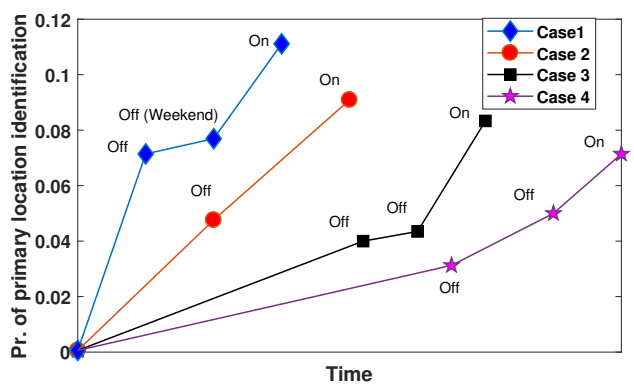

Figure 3: Figure illustrating the increase of probability of correctly identifying the primary locations given auxiliary information.

In all four cases, it was observed that a person's absence in the building during weekdays significantly reduces the size of the candidate set initially. This is due to two reasons. First, identifying the candidate set of locations that are occupied during weekdays will eliminate almost all sensors that are located in hallways, which are usually occupied during weekdays. Second, since the building chosen for this study does not allow working from home, being absent during weekdays is a rare event.

\section{MITIGATION STRATEGY}

In this section, we propose a set of potential mitigation strategies against the proposed attack.

\subsection{Principle of Least Privilege for Privacy}

Principle of least privilege has been widely accepted as one of the governing principles in designing mitigation strategies in security. The principle of least privilege states that the minimum amount of information and resources should be given to a subject in order for the subject to perform required duties. The same principle can be used for privacy enhancement as stated in [11].

For building applications, we need to ensure that only the minimum, necessary level of occupancy information is disclosed. For example, for occupancy-driven HVAC control, the controller only requires occupancy information at the zone level, but does not require the information at the individual sensor level [12]. 
The adversary could still potentially perform the attack using the zone level occupancy information to at least identify which zone includes the targeted individual's primary location. On the days when the individual is not present in the building, the adversary would expect to observe a decrease in the number of occupants in the zone. However, unlike the attack performed on the individual sensor level, the behaviors of other occupants in the zone would affect the accuracy of the identified sets in (3). It is possible that the decrease in the number of occupants could be due to other individuals not present in the building when the targeted individual is present. Similarly, even when the targeted individual is not present in the building, other individuals that were not previously in the zone could be present, resulting in no change or even an increase in the number of occupants in the zone. Moreover, even if the adversary were able to correctly identify the zone where the targeted individual resides in, $k$-anonymity [13] will be provided where $k$ is the number of sensors in the zone.

\subsection{Differential Privacy}

When the occupancy information at the zone level is released, it is possible to implement additional privacy enhancing mechanisms that will provide differential privacy. In this setting, probabilistic noise is added to the released occupancy information to ensure that answers to queries regarding the occupancy level in a zone would be statistically similar even when a single individual is present or not present in the zone [14].

We have implemented PeGaSus [9] on the collected occupancy data to examine how the utility of the occupancy data would change as we vary the parameter $\epsilon$. However, initial results suggest that even for a relatively large value of $\epsilon$, the utility of the gathered occupancy data decreases significantly. Finding the optimal tradeoff between the utility and privacy for the differential privacy based mitigation would be part of future work.

\subsection{Deletion of Past Information}

The proposed attack does not rely on the particular times when the presence data are collected. Whether the presence data was collected in the past or present, any data that matches the auxiliary information would be equally valuable in narrowing down the set of candidate sensor IDs of the targeted individual.

While the past data could be valuable in many applications that rely on predicting future occupancy levels as well as energy consumptions through machine learning techniques, keeping a large amount of past data will provide additional opportunities for the adversary. Setting an expiration time on the gathered occupancy data and either deleting or encrypting it automatically at the time of expiration will provide additional privacy against the proposed attack by eliminating the occupancy data that correspond to auxiliary information in the past.

\section{CONCLUSIONS}

In this paper, we studied privacy implications of occupancy sensors in smart buildings. We have proposed a privacy breaching attack in which the adversary combines the gathered occupancy data with auxiliary information, and identifies the sensor ID that is installed at the primary location of the targeted individual. We have validated the feasibility of the proposed attack using real-world data where we have demonstrated that even a small amount of auxiliary information is sufficient to greatly reduce the set of sensors that the targeted individual's sensor ID is located in.

We have also proposed mitigation strategies against the proposed attack. The principle of least privilege can be used as a governing principle in providing the right level of information to various applications. Differential privacy and deletion of past occupancy data could also be used to enhance the privacy of the building occupants. However, the exact details of implementing the mitigation strategies will differ from application to application. A systematic approach of determining the exact parameters of the mitigation strategies will be part of future work.

\section{ACKNOWLEDGEMENT}

This material is based on research sponsored by DARPA under agreement number FA8750-16-2-0021. The U.S. Government is authorized to reproduce and distribute reprints for Governmental purposes notwithstanding any copyright notation thereon. The views and conclusions contained herein are those of the authors and should not be interpreted as necessarily representing the official policies or endorsements, either expressed or implied, of DARPA or the U.S. Government.

\section{REFERENCES}

[1] Deborah Snoonian. Smart buildings. IEEE spectrum, 40(8):18-23, 2003.

[2] Antoni Martínez-Ballesté and et al. The pursuit of citizens' privacy: a privacyaware smart city is possible. IEEE Communications Magazine, 51(6):136-141, 2013.

[3] Ruoxi Jia and et al. Privacy-enhanced architecture for occupancy-based HVAC control. In ACM/IEEE 8th International Conference on Cyber-Physical Systems (ICCPS), pages 177-186. IEEE, 2017.

[4] Xiao Wang and Patrick Tague. Non-invasive user tracking via passive sensing: Privacy risks of time-series occupancy measurement. In Proceedings of the Workshop on Artificial Intelligent and Security Workshop, pages 113-124. ACM, 2014.

[5] Yuvraj Agarwal and et al. Occupancy-driven energy management for smart building automation. In Proceedings of the 2nd ACM workshop on embedded sensing systems for energy-efficiency in building, pages 1-6. ACM, 2010.

[6] Sharad Mehrotra and et al. Tippers: A privacy cognizant iot environment. In IEEE International Conference on Pervasive Computing and Communication Workshops (PerCom Workshops), pages 1-6. IEEE, 2016.

[7] Himanshu Khurana and Daniel Kirschner. Space utilization and building management system analysis, September 27 2018. US Patent App. 15/467,824.

[8] Primal Pappachan and et al. Towards privacy-aware smart buildings: Capturing, communicating, and enforcing privacy policies and preferences. In Distributed Computing Systems Workshops (ICDCSW), 2017 IEEE 37th International Conference on, pages 193-198. IEEE, 2017.

[9] Yan Chen and et al. PeGaSus: Data-adaptive differentially private stream processing. In Proceedings of the 2017 ACM SIGSAC Conference on Computer and Communications Security, pages 1375-1388. ACM, 2017.

[10] Sameera Ghayyur and et al. Iot-detective: Analyzing iot data under differential privacy. In Proceedings of the 2018 International Conference on Management of Data, pages 1725-1728. ACM, 2018.

[11] Lin Liu and et al. Security and privacy requirements analysis within a social setting. In Proceedings. 11th IEEE International Requirements Engineering Conference, 2003., pages 151-161. IEEE, 2003.

[12] Varick L Erickson and et al. Observe: Occupancy-based system for efficient reduction of hvac energy. In Proceedings of the 10th ACM/IEEE International Conference on Information Processing in Sensor Networks, pages 258-269. IEEE, 2011.

[13] Latanya Sweeney. k-anonymity: A model for protecting privacy. International fournal of Uncertainty, Fuzziness and Knowledge-Based Systems, 10(05):557-570, 2002.

[14] Cynthia Dwork. Differential privacy. Encyclopedia of Cryptography and Security, pages 338-340, 2011. 